\title{
Desenvolvimento e pós-colheita de abacaxi ornamental ${ }^{(1)}$
}

\author{
ROBSON ASSUNÇÃO CAVALCANTE², JOSÉ LUIZ MOSCA ${ }^{3}$, ALAN BERNARD OLIVEIRA DE SOUSA ${ }^{4}$, \\ DANIEL RODRIGUES CAVALCANTE FEITOSA ${ }^{4}$ e WALDELICE OLIVEIRA DE PAIVA ${ }^{3}$
}

\begin{abstract}
RESUMO
O presente trabalho foi dividido em duas etapas que tiveram como objetivos, avaliar a durabilidade pós-colheita de Ananas comosus var. erectifolius colhidos em três pontos de colheita, armazenados e refrigerados, bem como determinar o período compreendido entre a indução floral ao ponto de colheita. No primeiro experimento, os abacaxis ornamentais foram colhidos em área experimental instalada nas dependências da Embrapa Agroindústria Tropical, em Pacajus - CE. As hastes foram classificadas quanto ao comprimento da infrutescência (CI) em: infrutescências com $40 \pm 2 \mathrm{~mm}$ (ponto 1); $46 \pm 2 \mathrm{~mm}$ (ponto 2) e $51 \pm 2 \mathrm{~mm}$ (ponto 3 ). $O$ experimento foi conduzido em DIC em esquema fatorial $3 \times 2 \times 5$ sob armazenamento à seco dentro de caixas de papelão sob duas temperaturas $\left(9^{\circ} \mathrm{C}\right.$ e $\left.12^{\circ} \mathrm{C}\right) \mathrm{e}$ avaliadas qualitativamente em intervalos de dois dias, seguindo escala subjetiva de notas, variando de 3 (qualidade superior) a 0 (sem qualidade comercial). Dos tratamentos avaliados, as hastes que apresentaram maiores notas foram as colhidas no ponto 1, apresentando vida útil de 13 dias, independente da temperatura de armazenamento. No segundo experimento foram selecionadas plantas em fase vegetativa e, nestas, fora realizada a indução floral utilizando carbureto de cálcio na dose $0,5 \mathrm{~g} /$ litro de água. O desenvolvimento morfológico das hastes florais foi acompanhado observando os seguintes parâmetros: comprimento da coroa (CC), da infrutescência (CI) e do pedúnculo (CP); diâmetro da coroa (DC), da infrutescência (DI) e do pedúnculo (DP), até o momento em que a infrutescência atingiu o ponto de colheita estabelecido no primeiro experimento (infrutescência com comprimento média de $40 \pm 2 \mathrm{~mm}$ ). O período compreendido da indução floral ao ponto de colheita foi de 71 dias.
\end{abstract}

Palavras-chave: Ananas comosus var. erectifolius, ponto de colheita, temperatura de armazenamento, indução floral.

\section{ABSTRACT \\ Development and post harvest of ornamental pineapple}

The present work was divided in two stages that had as objectives to evaluate the durability post harvest of Ananas comosus var. erectifolius harvested in three stages, stored and cooled, and to determine the period required from floral induction to the harvest stage. In the first experiment, the ornamental pineapple had been harvested in experimental area installed at Embrapa Tropical Agroindustry in Pacajus - Ceará state - Brazil. The floral stems had been classified by the length of infrutescence (CI) in 3 harvest stages: infrutescences

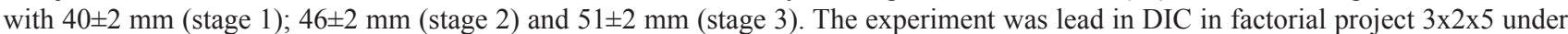
two temperatures $\left(9^{\circ} \mathrm{C}\right.$ and $12^{\circ} \mathrm{C}$ ), being evaluated qualitatively every two days, following note scale varying of 3 (superior quality) the 0 (without commercial quality). The higher notes were observed at stage 1, which showed useful life of 13 days, regardless of the temperature of storage. In the second experiment, plants in vegetative stage were selected, and these had been performed using the floral induction of calcium carbide in the dose $0.5 \mathrm{~g} /$ liter of water. The morphological development of the floral stems was observed by the following parameters: height of the infrutescence (HF), height of the crown (HC), diameter of the infrutescence (DF), diameter of the crown (DC), diameter of the peduncle (DH) and length of the peduncle $(\mathrm{CH})$, until the moment where the infrutescence reached the point of harvest established in the first experiment (infrutescence with average height of $40 \pm 2 \mathrm{~mm}$ ). The period from the floral induction to the harvest point was of 71 days.

Keywords: Ananas comosus var. erectifolius, postharvest, harvest stage, storage temperature, floral induction.

\section{INTRODUÇÃO}

A família Bromeliaceae é representada por espécies de grande importância ornamental e econômica, com algumas plantas que, além de muito usadas em paisagismo de ambientes, apresentamse como fornecedoras de fibras e frutos (REINHARDT, 2000). Dentre as espécies de destaque temos a Ananas comosus L. (abacaxi comestível) que, segundo a atual classificação botânica proposta por D'EECKENBRUGGE e LEAL (2003), foram adicionadas algumas variedades ornamentais antes classificadas como espécies distintas, isto devido a semelhanças morfológicas entre outras características de ordem fisiológicas e moleculares. Dentre essas novas variedades encontra-se Ananas comosus var. erectifolius, antes classificada como A. lucidus Miller., caracterizada pela coroa sem espinhos, infrutescências de coloração avermelhada que chegam a medir $10 \mathrm{~cm}$ de comprimento dispostas na posição apical do pedúnculo de até $80 \mathrm{~cm}$ de comprimento (BORGES, 2000).

Segundo BORGES (2000), o abacaxi ornamental é exportado principalmente para os Estados Unidos, Holanda, Alemanha e Inglaterra por alguns produtores localizados no estado do Ceará, tendo o estado movimentado no ano de 2003

\footnotetext{
${ }^{1}$ Recebido para publicação em 19/05/2008 e aprovado em 03/05/2010.

${ }^{2}$ Universidade Federal do Ceará, Mestrando em Fitotecnia, Fortaleza, CE, agrobson@gmail.com.

${ }^{3}$ Embrapa Agroindústria Tropical, CP 3761, 60511-110, Fortaleza, CE, mosca@cnpat.embrapa.br

${ }^{4}$ Universidade Federal do Ceará, Graduando em Agronomia, Fortaleza, CE.
} 
em torno de US\$ 298,9 mil com as exportações (BAIMA, 2004 citado por CARVALHO et al. 2009). Isto mostra que este produto apresenta potencial comercial, conferido principalmente pela sua exoticidade, e pode apresentar como alternativa para incrementar a comercialização da floricultura de flores de corte para buquês que, segundo KIYUNA et al.(2008), apresentou em 2007 decréscimo no volume exportado pelo Brasil.

Para que o abacaxi ornamental oferecido ao mercado ganhe cada vez mais espaço no cenário internacional é preciso que este apresente qualidade superior e a pesquisa precisa estar presente em todas as etapas do sistema produtivo, para que se conheça melhor o comportamento desta cultura e que se desenvolvam novas tecnologias para fornecer produto que satisfaça não só o comprador, mas também as necessidades do produtor.

$\mathrm{Na}$ cultura do abacaxi ornamental diversos são os trabalhos na área de propagação, principalmente cultura de tecidos onde se busca formular protocolos de produção de mudas de qualidade a custo mais baixo, porém, ainda há poucos trabalhos em outras áreas, tornando restritas informações sobre cultivo, manejo e pós-colheita.

Com base no exposto, o presente trabalho teve como objetivo avaliar a durabilidade pós-colheita de Ananas comosus colhidos em três pontos de colheita distintos, armazenados e refrigerados, bem como determinar o período compreendido entre a indução floral e a colheita.

\section{MATERIAL E MÉTODOS}

O experimento foi realizado em duas etapas, sendo a primeira para verificar o melhor ponto de colheita hastes florais de abacaxi ornamental (Ananas comosus var. erectifolius) conforme a durabilidade pós-colheita dos materiais selecionados e, a segunda etapa, realizar o acompanhamento do desenvolvimento fenológico da infrutescência até o ponto de colheita estabelecido anteriormente.

Foram utilizadas plantas de abacaxi ornamental, provenientes de mudas micropropagadas em laboratório, com três anos de estabelecimento na área. Estas plantas foram cultivadas em casa de vegetação situada no campo experimental da Embrapa Agroindústria Tropical localizado a $4^{\circ} 10^{\prime} \mathrm{S}$ e $38^{\circ}$ $27^{\prime}$ W, referente ao município de Pacajus - Ceará (temperatura média de $30^{\circ} \mathrm{C}$ ) e plantadas em filas simples, com espaçamento $0,5 \mathrm{~m} \times 0,5 \mathrm{~m}$.

Na primeira etapa foi realizada colheita manual com auxílio de objeto cortante em todas as plantas de abacaxi ornamental que apresentavam frutificação. Após a colheita foi realizada a seleção das hastes florais, aqui definido como o conjunto constituído pela coroa, infrutescência e pedúnculo, de acordo com a comprimento da infrutescência, sendo classificadas em três pontos de colheita: $40 \pm 2 \mathrm{~mm}, 46 \pm 2 \mathrm{~mm}$ e $51 \pm 2 \mathrm{~mm}$, sendo denominados ponto 1,2 e 3 , respectivamente. Após a colheita e seleção, o material foi conduzido para o Laboratório de Fisiologia e Tecnologia Pós-colheita da Embrapa, localizado em Fortaleza, sendo este transporte realizado em automóvel com as hastes florais condicionadas em caixas de papelão (dimensões: $1 \mathrm{~m}$ de comprimento, 0,32 $\mathrm{m}$ de largura e 0,20 $\mathrm{m}$ de comprimento) e sem hidratação.

No laboratório, o material foi submetido ao procedimento de limpeza, que consistiu em remoção manual dos vestígios florais presentes na infrutescência, lavagem em jato d'água, conforme utilizados por produtores no estado do Ceará para retirada de pó presente na haste floral, e secagem sob hidratação à temperatura ambiente $\left(25^{\circ} \mathrm{C}\right)$.

As hastes foram armazenadas em ambiente refrigerado a $9^{\circ} \mathrm{C}$ e $12^{\circ} \mathrm{C}$ dentro de caixas tampadas, sendo que as quais apresentavam dimensões semelhantes às utilizadas no transporte do campo ao laboratório sendo as hastes avaliadas quanto a qualidade visual a cada dois dias até o momento em que todos os tratamentos apresentassem elevado grau de senescência, segundo escala subjetiva de notas:

3 - Coroa, infrutescência e pedúnculos sem sinais de ressecamento ou mudança de cor características,

2 - Início de ressecamento na coroa e/ou pedúnculo, leve alteração na cor e brilho da infrutescência,

1 - Ressecamento na coroa e/ou pedúnculo, alteração acentuada na cor e brilho da infrutescência,

0 - Sinais de senescência acentuados, separação da infrutescência do pedúnculo.

Nesta etapa, o experimento foi conduzido delineamento inteiramente casualizados em esquema fatorial 3 (ponto de colheita) x 2 (condição de armazenamento) com 5 repetições, constituídas por uma haste.

A segunda etapa foi realizada tão logo se obteve o resultado da primeira etapa. Na mesma área onde foram retiradas as infrutescências para o teste pós-colheita, no final do mês de janeiro foram selecionadas as plantas que estavam na fase reprodutiva para a indução floral (solução formada por $50 \mathrm{~g}$ de carbureto de cálcio diluído em 10 litros de água), cada planta recebendo aproximadamente $50 \mathrm{ml}$ da solução no centro da roseta.

Após a indução floral foi realizado acompanhamento periódico para identificação do início da "floração" e o momento que as hastes florais apresentavam a infrutescência e coroas que pudessem ser mensuradas.

A partir de 50 dias após a indução floral, foram identificadas 50 plantas e, nestas, acompanhou-se o crescimento médio da comprimento da infrutescência e da coroa, diâmetro da infrutescência e da coroa, diâmetro e comprimento do pedúnculo, conforme ilustrado na figura $1 \mathrm{em}$ intervalos de 7 dias entre as determinações até o momento em que as infrutescências apresentassem o ponto de colheita estabelecido na etapa anterior. Nesta segunda etapa, trabalhou-se com os valores médios sendo em seguida determinadas as curvas de crescimento até o ponto de colheita.

\section{RESULTADOS E DISCUSSÃO}

Independente da temperatura de armazenado, apenas o ponto de colheita influenciou na manutenção da qualidade do abacaxi ornamental. Embora não sendo objeto de estudo nesse trabalho, o armazenamento refrigerado pode induzir a injúrias após a alteração da condição conforme mostrado por SOUTO et al (2004) em abacaxi 'Pérola' que, após a retirada do armazenamento refrigerado a $8^{\circ} \mathrm{C}$, verificaram sinais de injúria pelo frio na infrutescência, o que, embora a temperatura não tenha se mostrado fator limitante para o abacaxi ornamental neste experimento, cabe a realização de futuros trabalhos 
específicos para a verificação de possíveis injúrias.

A figura 2 mostra que hastes colhidas no ponto mais prematuro tiveram, segundo a escala subjetiva de notas, maiores notas durante o período de observação, seguidas das hastes nos pontos 2 e 3 que apresentaram degradação mais acelerada. No ponto de colheita 1 o momento do descarte, caracterizado quando os abacaxis ornamentais apresentavam nota com média inferior a 1 , foi adiado sendo descartada no $13^{\circ}$ dia, enquanto os demais tratamentos apresentavam tal classificação no $11^{\circ}$ dia de armazenamento. Segundo CHITARRA e CHITARRA (1990) o estádio de maturação há importante influencia na vida útil póscolheita dos produtos vegetais, podendo colheitas realizadas sem a completa maturação fisiológica afetar a qualidade durante o armazenamento, assim como em grau de maturação mais avançada reduzir a vida útil por apresentar processos fisiológicos mais acelerados com alta intensidade respiratória (aumento na quantidade de calor gerado), além de apresentar maior sensibilidade a danos físicos levando à perdas quantitativas e qualitativas (CHITARRA, 1998); (FONSECA et al. (2002).

A época do ano que é realizada a indução floral influi diretamente no período compreendido entre a aplicação do tratamento e o momento da colheita, é o que revela o trabalho de CARVALHO et al (2005) onde constataram que abacaxizeiros 'Smooth Cayenne' com indução floração utilizando ethephon nos meses de abril, maio, junho e julho apresentaram ciclos diferenciados e frutos distintos também em relação à qualidade. O abacaxizeiro ornamental completou o ciclo produtivo no final de janeiro, 71 dias após a indução floral, logo, sugerimos novos testes com indução em outros meses do ano para verificar se há diferenciação nos resultados.

Após a indução floral nas plantas, transcorreram 50 dias para que o acompanhamento semanal do crescimento da região reprodutiva da planta fosse iniciado, no entanto, neste momento o pedúnculo já estava desenvolvido, mostrando ser a estrutura que apresenta desenvolvimento inicial mais pronunciado, média de $40 \mathrm{~cm}$ de comprimento e $8 \mathrm{~mm}$ de diâmetro. $\mathrm{O}$ pedúnculo apresentou incremento no comprimento de $8 \mathrm{~cm}$ do $50^{\circ}$ ao $58^{\circ}$ dia com redução da taxa de crescimento nas avaliações posteriores, $2 \mathrm{~cm} \mathrm{e} 1 \mathrm{~cm} \mathrm{no} 64^{\circ}$ e $71^{\circ}$ dia, respectivamente (figura $3)$. No diâmetro a taxa de crescimento foi menor, estabilizandose já a partir do $54^{\circ}$ dia após a indução.

O crescimento horizontal e vertical da infrutescência foi observado nas primeiras observações (figura 4), com as curvas de desenvolvimento da infrutescência apresentando comportamento semelhante, mas mostrando $\mathrm{o}$ fator comprimento sempre superior, comportamento explicado pelas evidentes características da estrutura reprodutiva.

No desenvolvimento da região da coroa na infrutescência, esta iniciou apresentando diâmetro maior que o comprimento, porém, embora os dois parâmetros apresentarem taxa de crescimento contínuo, o desenvolvimento em diâmetro foi superado pelo do comprimento a partir do $60^{\circ}$ dia após a indução floral.

\section{CONCLUSÕES}

Nas condições deste experimento conclui-se que o ponto de colheita mais recomendado entre os avaliados é quando a infrutescência apresenta $40 \pm 2 \mathrm{~mm}$ de comprimento.

$\mathrm{O}$ ciclo apresentado do momento da indução floral à colheita em abacaxizeiro ornamental para o ponto de colheita determinado é de 71 dias.

\section{REFERÊNCIAS}

BORGES, N.S.S. Influência da adição de meio de cultura líquido no crescimento e desenvolvimento de gemas de abacaxi ornamental (Ananas lucidus Miller). Fortaleza: Centro de Ciências Agrárias, UFC, 2000, 44p. Monografia (Graduação em Agronomia).

CARVALHO, S.L.C.; NEVES, C.S.V.J.; BÜRBLE, R.; MARUR, C.J. Épocas de indução floral e soma térmica do período de florescimento à colheita de abacaxi 'Smooth Cayenne'. Revista Brasileira de Fruticultura, Jaboticabal - SP, v.27, n.3, p.430 - 433, dez. 2005.

CARVALHO, A.C.P.P.; PINHEIRO, M.V.M.; DIAS, G. de M.G. e MORAIS, J.P.S. In vitro multiplication of ornamental pineapple by shoot etiolation and regeneration. Horticultura Brasileira. vol.27, n.1, 2009.

CHITARRA, M.I.F. Processamento mínimo de frutos ehortaliças. Viçosa: Centro de Produções Técnicas, 1998. 88 p.

CHITARRA, M.I.F.; CHITARRA, A.B. Pós-colheita defrutos e hortaliças: fisiologia e manuseio. Lavras: ESAL-FAEPE, 1990. $320 \mathrm{p}$.

D’EECKENBRUGGE, G. C.; LEAL, F. Morphology, anatomy and taxonomy. In: BARTHOLOMEW, D.P.; PAULL, R.E.; ROHRBACH, K.G. (Eds.). The pineapple: botany, production, and uses. New York: CAB International, p. 13-32, 2003.

FONSECA, S.C.; OLIVEIRA, F.A.R.; BRECHT, J.K. Modelling respiration rate of fresh fruits and vegetables for modified atmosphere packages: a review. Journal of Food Engineering, Kidlington, v.52, n.1, p.99-119, 2002.

KIYUNA, I.; ÂNGElO, J. A.; COELHO, P. J. Flores: desempenho do comércio exterior em 2007. Análise e indicadores do agronegócio. Instituto de economia agrícola, v.3, n.1, janeiro de 2008 .

REINHARDT, D. H.; SOUZA, L. F. S.; CABRAL, J. R. S. Abacaxi. Produção: aspectos técnicos. Embrapa Mandioca e Fruticultura - Cruz das Alamas, BA. p.77, il; (Frutas do Brasil; 7), 2000.

SOUTO, R.S.; DURIGAN, J.F.; SOUZA, B.S.; DONADON, J.; MENEGUCCI, J.L.P. Conservação pós-colheita de abacaxi 'PÉROLA' colhido no estádio de maturação "pintado" associando-se refrigeração e atmosfera modificada. Revista Brasileira de Fruticultura, Jaboticabal - SP, v.26, n.1, p.24 -28 , abr. 2004. 


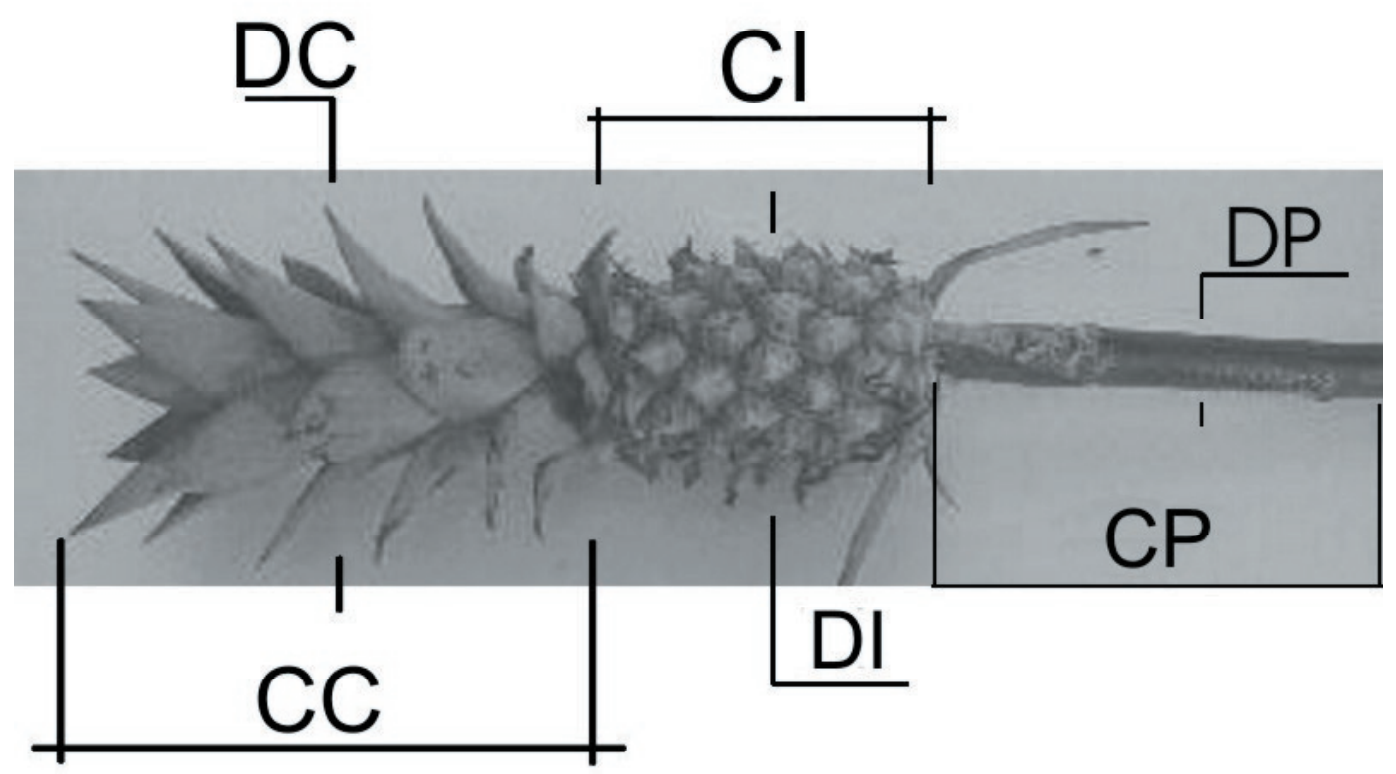

Figura 1. Esquema de parâmetros avaliados durante desenvolvimento da haste floral: comprimento da coroa (CC), da infrutescência $(\mathrm{CI})$ e do pedúnculo $(\mathrm{CP})$; diâmetro da coroa (DC), da infrutescência (DI) e do pedúnculo (DP).

Figure 1. Parameters evaluated during development of the flower stems: lenght of the crown (CC); infrutescence (CI) and peduncle (CP) and, diameter of the crown (DC), infrutescence (DI) and peduncle (DP).

\section{Ponto $1 \quad$ a Ponto $2 \quad \Delta$ Ponto 3}

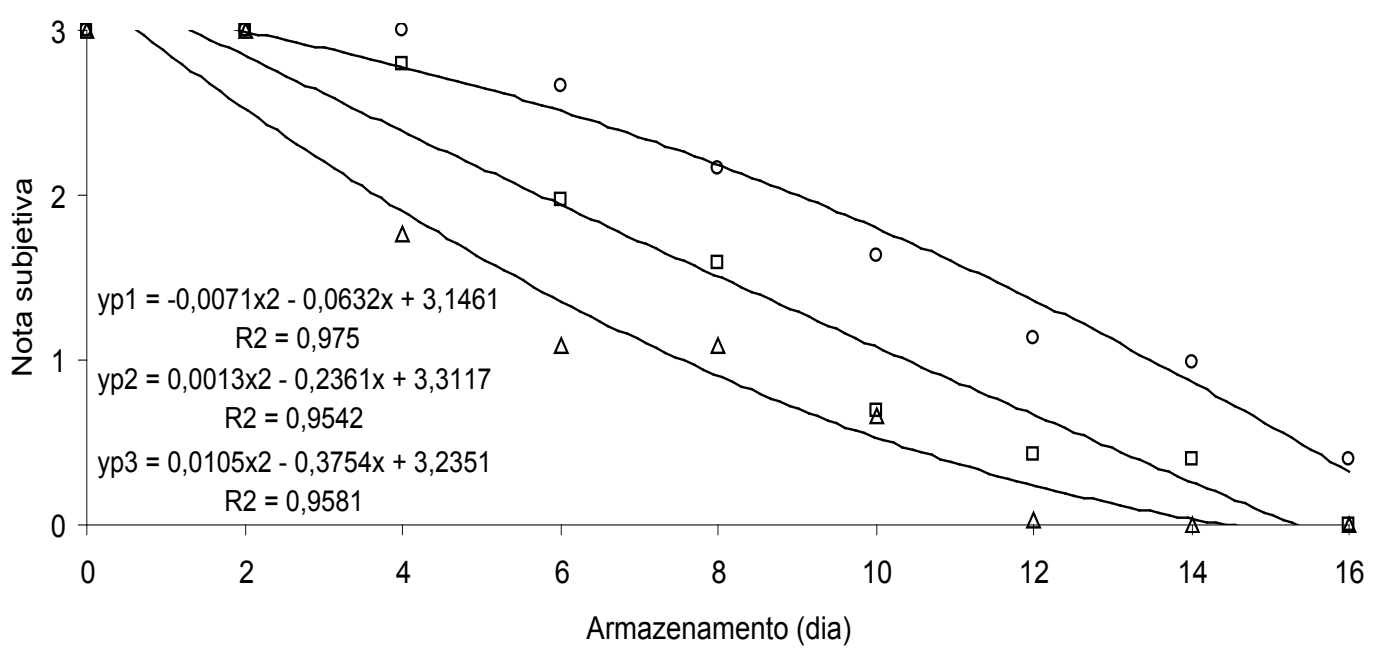

Figura 2. Notas da avaliação qualitativa de hastes florais de abacaxi ornamental colhidos em três pontos de colheita (ponto 1: infrutescência com $40 \pm 2 \mathrm{~mm}$ de comprimento, ponto 2: $46 \pm 2 \mathrm{~mm}$ e ponto 3: $51 \pm 2 \mathrm{~mm}$ ) independentemente do ambiente. Figure 2. Notes to the qualitative evaluation of floral connecting rods of ornamental pineapple harvested in 3 different points of harvest (point 1: infrutescência with $40 \pm 2 \mathrm{~mm}$ of lenght, point 2: $46 \pm 2 \mathrm{~mm}$ and point 3: $51 \pm 2 \mathrm{~mm}$ ) independently of the environment. 


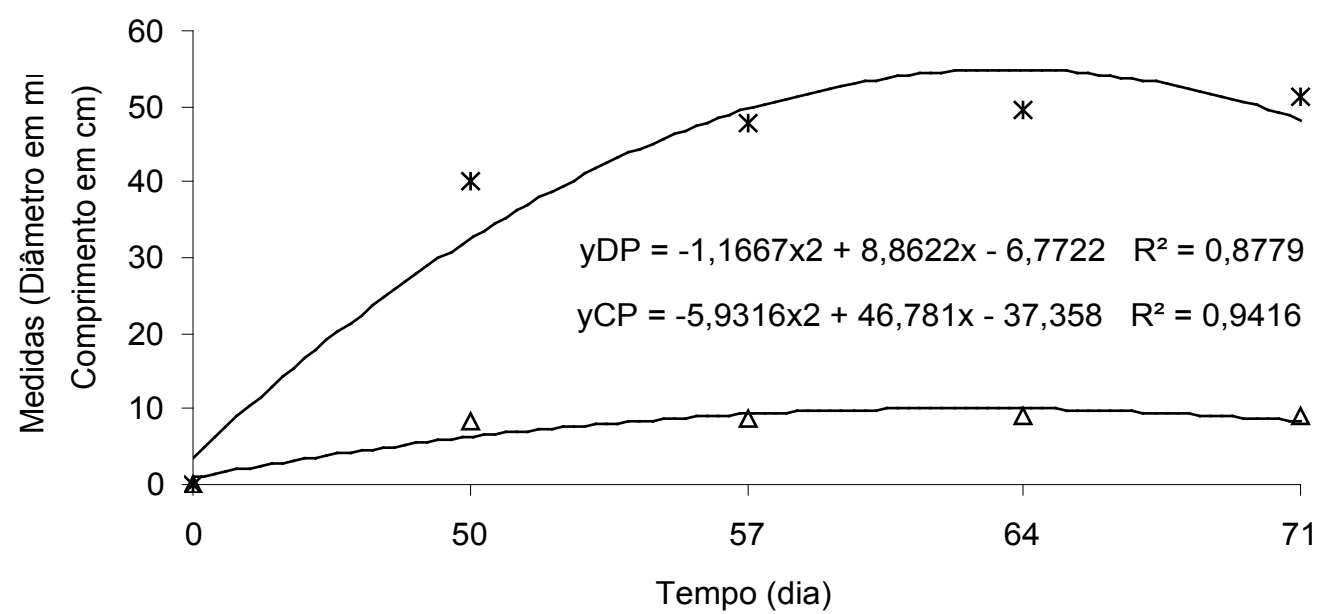

Figura 3. Diâmetro e comprimento do pedúnculo floral de abacaxi ornamental, período compreendido entre a indução floral e o ponto de colheita.

Figure 3. Diameter and length of the floral peduncle of ornamental pineapple, period required from floral induction to the harvest stage.

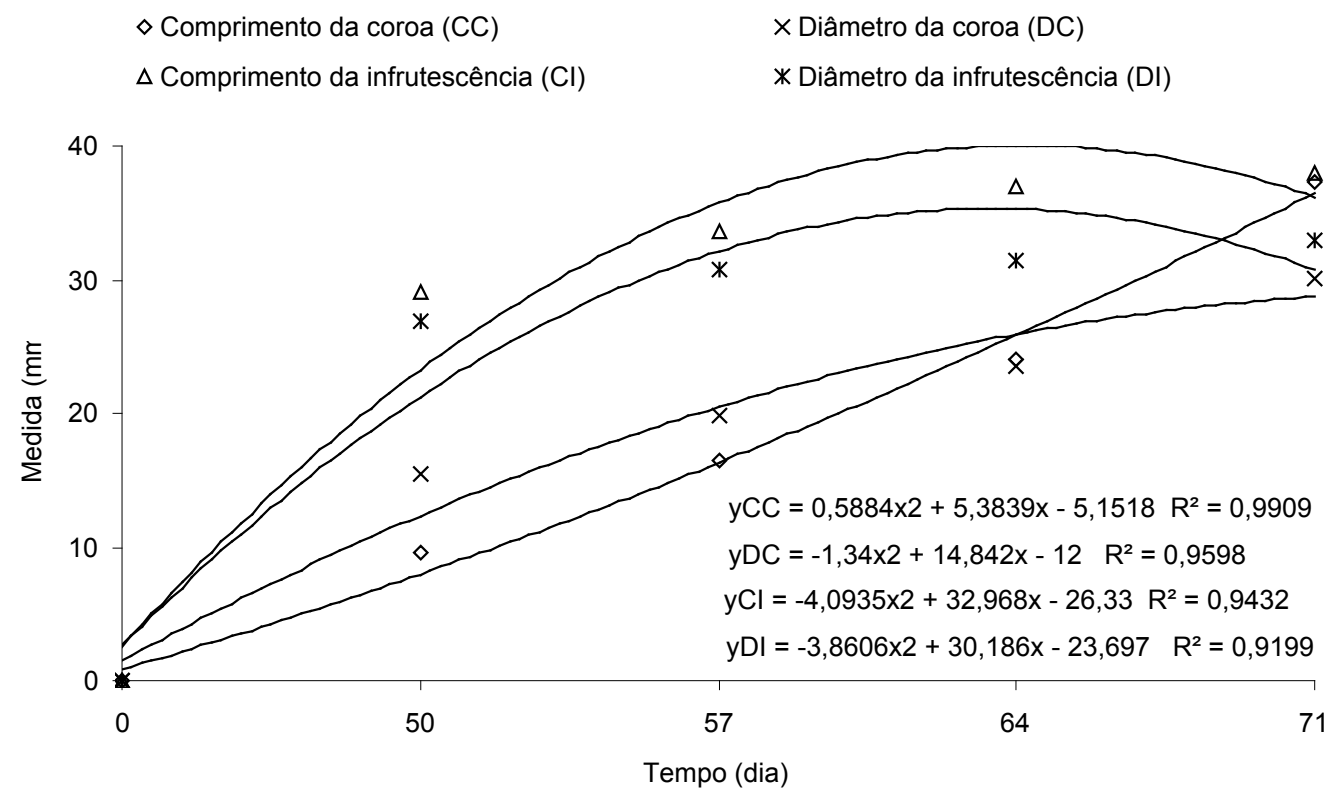

Figura 4. Diâmetro e comprimento da coroa e infrutescência de abacaxi ornamental, no período compreendido entre a indução floral e o ponto de colheita.

Figure 4. Diameter and length of the crown and infrutescence of ornamental pineapple, during the period required from floral induction to the harvest stage. 